\title{
Cavitation Status Recognition Method of Centrifugal Pump Based on Multi-Pointand Multi-Resolution Analysis
}

\author{
L. Dong ${ }^{1,2}$, J. C. Zhu ${ }^{1}$, K. Wu ${ }^{1}$, C. Dai ${ }^{3}$, H. L. Liu ${ }^{1 \dagger}$, L. X. Zhang ${ }^{1}$, J. N. Guo ${ }^{1}$ and H. B. \\ $\operatorname{Lin}^{2}$ \\ ${ }^{1}$ Research Center of Fluid Machinery Engineering and Technology, Jiangsu University, Zhenjiang ,Jiangsu \\ Province, 212013, China \\ ${ }^{2}$ Sichuan Provincial Key Lab of Process Equipment and Control, Sichuan University of Science \& \\ Engineering, Zigong, Sichuan Province, 643000,China \\ ${ }^{3}$ School of Energy and Power Engineering, Jiangsu University, Zhenjiang, Jiangsu Province, 212013, China \\ †Corresponding Author Email: liuhoulin@ujs.edu.cn
}

(Received March 15, 2020; accepted May 29, 2020)

\begin{abstract}
Cavitation monitoring is particularly important for pump efficiency and stability. It is easy to misjudge cavitation by using a given threshold of a single eigenvalue. In this work, based on the vibration signal, a method for multi-resolution cavitation status recognition of centrifugal pump is proposed to improve the accuracy and universality of cavitation status recognition., wavelet packet decomposition (WPD) is used to extract the statistical eigenvalues of multi-scale time-varying moment of cavitation signal after reducing the clutter, such as root mean square value, energy entropy value and so on. The characteristic matrix is constructed. Principal component analysis method (PCA) is employed to reduce the dimension of the characteristic matrix and remove the redundancy, which constructs the radial basis function (RBF) neural network as the input. The results show that the overall recognition rate of non-cavitation, inception cavitation and serious cavitation by using the vibration signal of one measuring point is more than $97.7 \%$. The recognition rate of inception cavitation is more than $80 \%$. Based on the vibration signal information fusion method of two measuring points, the recognition rate of centrifugal pump inception cavitation status reaches more than $99 \%$, and the recognition rate of vibration signal information fusion method of three measuring points reaches $100 \%$ for all three cavitation statuses. Due to the influence of factors such as change of external excitation and abrupt change of working conditions, sensor data acquisition is often subjected to unpredictable disturbance. To study the ability of single-point cavitation status recognition method to resist unknown disturbances, by constantly adjusting the value of the interference coefficient of the interference term. It is found that the recognition rate of cavitation status using single measuring point decreases almost linearly with the increase of the interference coefficient. When five measuring points are used for information fusion cavitation status recognition, the cavitation status recognition rate still reaches over $90 \%$ even if the interference factor of one measuring point reaches $50 \%$.
\end{abstract}

Keywords: Centrifugal pump; Cavitation recognition; Vibration; Wavelet packet decomposition; Principal component analysis; RBF neural network.

\section{INTRODUCTION}

As the most widely used general machinery, the pump plays a key role in the fields of hydraulic transportation, water irrigation, and hydropower generation. However, the cavitation within a pump has restricted the development of the pump industry. The cavitation can appear during the entire range of operating conditions in pumps. The generation and development of cavitation in the pump can change the velocity distribution in the flow channel, which is resulted in the decrease of energy characteristics of the pump. The development of cavitation will destroy the flow passage to a certain extent, and even cause the vibration of pump. Therefore, it is of great significance to study the phenomenon of cavitation in the pump.

Cavitation signals are complex, coupled and uncertain, the traditional performance parameter 
method considers the centrifugal pump head drop of $3 \%$ as the judgment basis for the inception cavitation of the centrifugal pump, which has hysteresis. Therefore, it is necessary to determine the cavitation monitoring signal and eigenvalues of the centrifugal pump with higher sensitivity (Samanipour et al. 2017). Wang and Chen (2009) presented a method for a centrifugal pump based on vibration signals. In the method, a wavelet transform was used to extract features from measured vibration signals, and a linearized neural network was used to distinguish cavitation types. Sakthivel et al. (2014) presented that the vibration signal was used to monitor the working condition of the single block centrifugal pump. In the paper dimensionality reduction is performed using traditional dimensionality reduction techniques and nonlinear dimensionality reduction techniques. By comparing different classifiers, a better combination method of dimension reduction classifiers is proposed. Alfayez et al. (2005) [4] presented a case study. The acoustic emission was applied for identifying the inception cavitation and determining the best efficiency point (BEP) of a $60 \mathrm{~kW}$ centrifugal pump, which had shown to offer early recognition of incipient cavitation. Farokhzad and Ahmadi (2013) concentrated on a procedure for prediction of cavitation using acoustic signals and multi-layer perceptron (MLP) neural network, and proved itself a fast and strong technique for frequency analysis in the steady conditions and constant speed. Durocher and Feldmeier (2004) used stator currents to recognize cavitation status in centrifugal pump. The approach might rely on noninvasive monitoring of three-phase induction motor to report equipment conditions and enable maintenance intervention before a fault occurs. Hernandez-Solis and Carlsson (2010) measured motor current and voltage signals for different operating points of the pump, and studied the correlation between the cavitation phenomena and the power of the motor. Which verifies the reliability of recognizing cavitation by the Motor Current and Power Signature Analyses (PSA)

There is no obvious demarcation between different cavitation statuses, which makes it difficult to set an accurate threshold according to the mapping relationship between cavitation statuses and eigenvalues. Moreover, most of the failures of the centrifugal pump or the change of the working conditions can cause changes in the eigenvalues such as the head, sound pressure level (SPL) and vibration level. As a result, it is easy to misjudge cavitation recognition by means of a single eigenvalue given threshold, which brings great challenges to the feature extraction and pattern recognition of centrifugal pump cavitation statuses. Bourdon et al. (1999) adopted vibration acceleration sensor to collect cavitation signals of hydraulic turbines, and extracted characteristic values to recognize cavitation and cavitation erosion statuses by statistical analysis and spectrum analysis. Rus et al. (2007) measured the vibration signals of axial flow turbines under cavitation conditions, which proved the correlation between the cavitation statuses and vibration. Yagi et al.
(2003) used vibration accelerometers to obtain signals at different cavitation statuses. It is believed that the peak frequency of the cavitation inception status signal focus on $20 \sim 30 \mathrm{kHz}$, and the frequency band is used as the basis for judging the inception of cavitation. Escaler et al. (2002) conducted a resonance test on the shrinkage model of a Francis turbine under different operating conditions and found that the cavitation signal focuses on the frequency range of $10 \sim 100 \mathrm{kHz}$. And the 10 15 $\mathrm{kHz}$ component was suitable as the characteristic frequency band. In addition, he proposed that different cavitation types required different measuring points arrangement and statuses recognition methods, and the attached cavitation measuring points should be as close to the impeller as possible. The characteristic frequency band is 10 $\mathrm{kHz}$. Cavitation is related to radial vibration of bearing, and the characteristic frequency band is about $3 \mathrm{kHz}$. The characteristic frequency band of vortex cavitation is $30 \sim 40 \mathrm{kHz}$. Dong et al. (2012). filtered the vibration signal of centrifugal pump and calculated the wide frequency vibration level. The basis of cavitation incipient was proposed. When the vibration signal is in the frequency band of 10 $50 \mathrm{~Hz}$, the broadband vibration level is $1 \%$ higher than that in the non-cavitation status, and when the vibration signal is in the frequency band of 1000$3000 \mathrm{~Hz}$, the broadband vibration level is $1.1 \%$ higher than that in the non-cavitation status, which can be considered as the inception cavitation. Xue. (2016) used multifractal algorithm to extract the eigenvalues of the natural mode function components of vibration signals to form the eigenvector as input, and constructed back propagation (BP) neural network for turbine fault pattern recognition. Asish et al. (2018) extracted several statistical features of the triaxial acceleration sensor data of pump and bearing shell, and input to support vector machine (SVM) algorithm for training and testing. So that the bubbles to be formed can be accurately identified at a higher rotation speed. Azizi et al. (2017) extracted several statistical features from the first six intrinsic mode functions (IMFs) components of empirical mode decomposition and established the generalized regression neural network (GRNN), obtaining the accuracy of cavitation status recognition reaching 97.5\%. Xue et al. (2013) extracted pump cavitation eigenvalue from the optimal frequency region of vibration signals collected, and obtained the optimal classification hyperplane for distinguishing cavitation statuses by SVM.

In summary, how to recognize the cavitation status of the pump more effectively is still a problem. Eigenvalues (such as mean, root mean square and standard deviation) are widely used in feature extraction. However, cavitation characteristics are distributed in a wide frequency range, and the characteristics of different frequency bands are also different. The use of single eigenvalues in the whole frequency band or a single characteristic frequency band cannot reflect the variation law of signals in different frequency bands before and after cavitation, and it is difficult to determine the 
eigenvalue thresholds that take into account different operating conditions. In addition, due to the influence of external harsh environment, excitation change and working condition mutation, sensor data acquisition is often subject to unpredictable disturbance, which is resulted in frequent mutation or loss of original data. So, higher requirements for the anti-interference of cavitation status recognition method undoubtedly need put forward.

A multi-point and multi-resolution recognition method for cavitation status of centrifugal pump based on vibration signal is proposed. Firstly, the WPD is used to extract the frequency band characteristics of decomposition. The PCA is adopted to reduce the dimension of the system and eliminate the redundancy of the system (Dong et al. 2019). Then, three cavitation statuses are recognized by using RBF neural network. Finally, the recognition effect and anti-interference of the three cavitation statuses of centrifugal pump are analyzed.

\section{Signal Processing Method}

To improve the recognition accuracy and efficiency of cavitation status for centrifugal pump, a cavitation recognition method based on WPD, PCA and RBF neural network are proposed. The structure of the proposed method is presented in Fig.1.

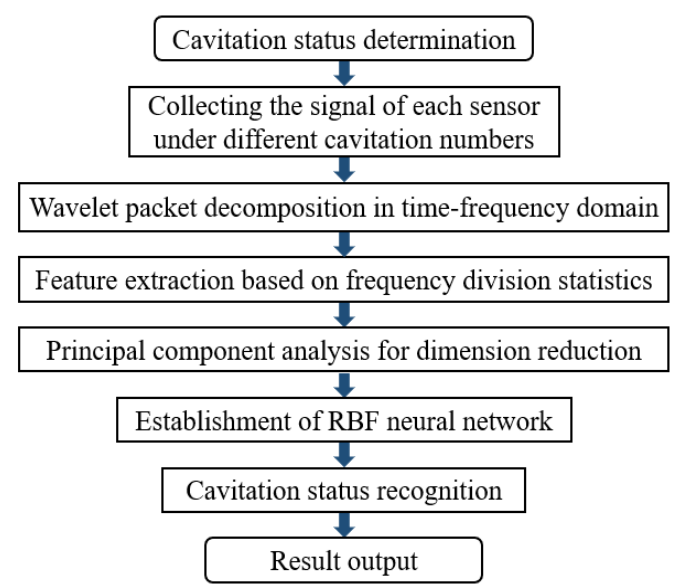

Fig. 1. Flowchart of cavitation recognition method.

The algorithmic for each major step in the method is as follows:

(1) Three cavitation statuses are demarcated by cavitation performance test.

(2) Signal of each sensor under different cavitation statuses is collected.

(3) The wavelet packet decomposition of each sensor signal is carried out to analyze its variation rule in time-frequency domain.
(4) The eigenvalues of nodes with larger energy coefficients are extracted to form the eigenvalue matrix.

(5) The PCA is used to reduce the dimension of the eigenvalue matrix to obtain the principal component matrix.

(6) Due to the large numerical difference between the eigenvalue elements, in order to eliminate redundancy and improve recognition accuracy and speed, the eigenvalue matrix is normalized. And the PCA is used to reduce the dimension to obtain a principal component matrix.

(7) The principal component matrix of training samples is input into RBF neural network as the input layer, and three cavitation statuses are used as the output layer to determine the number of nodes in the hidden layer and the structure of RBF neural network. After the training samples are fully trained and meet the network accuracy requirements or reach the number of iterations, an ideal RBF neural network is obtained.

(8) The principal component matrix of the test sample is input into the ideal RBF neural network to get the cavitation recognition results.

\section{Cavitation DETERMINATION}

StatuS

Johann (2014) pointed out that the (NPSH) 1 (head drop of $1 \%$ ) and (NPSH) 3 (head drop of 3\%) can be used as standards for recognizing the cavitation status in pumps. Based on these, the whole cavitation process is normally divided into three statuses (He et al. 2017): the non-cavitation status (head drop is less than 1\%), the inception cavitation status (head drop is between $1 \%$ and 3\%) and the serious cavitation status (head drop is higher than $3 \%$ ). To accurately obtain the cavitation numbers corresponding to $1 \%$ and $3 \%$ drop of head, the multi-measurement method was adopted during the test. Eventually, the average cavitation performance curves of the centrifugal pump under three typical flow rates were obtained, as shown in Fig. 2. The corresponding cavitation numbers for different status were shown in Table 1 . The cavitation numbers defined by Eq. (1),

$$
\sigma=\frac{P_{i n}-P_{v}}{0.5 \rho V_{0}^{2}}
$$

Here $\sigma$ is Cavitation number under different operating status, $P_{\text {in }}$ is Pump inlet pressure, $P_{v}$ saturated vapor pressure of a medium at ambient temperature (The value of $P_{v}$ in the manuscript is $3,574 \mathrm{pa}), \rho$ is the density of the medium, $P_{v}$ is the flow rate of the medium. 
Table 1 Cavitation numbers for different cavitation statuses

\begin{tabular}{|c|c|c|c|}
\hline Flow rate $/\left(\mathrm{m}^{3} / \mathrm{h}\right)$ & Non-cavitation & Inception cavitation & Serious cavitation \\
\hline 10 & & & \\
\hline 12.5 & $>0.020$ & $0.018-0.020$ & $<0.018$ \\
\hline 15 & $>0.022$ & $0.019-0.022$ & $<0.019$ \\
\hline
\end{tabular}

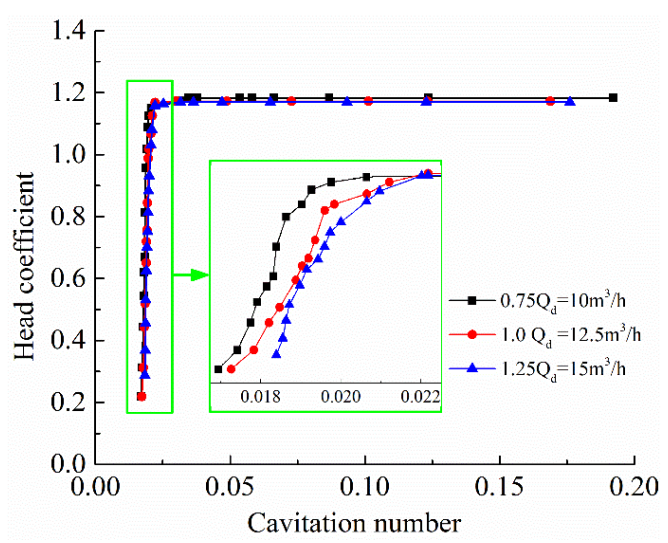

Fig. 2. Cavitation performance curves under three flow rates.

\section{Cavitation Status Recognition}

\subsection{Wavelet Packet Decomposition in Time-Frequency Domain}

The frequency spectrums of cavitation vibration under different cavitation statuses are mainly characterized by broadband. Simply studying the amplitude at certain characteristic frequencies or the overall energy value cannot accurately reflect the characteristics of cavitation. Therefore, it is of great significance to study the time-frequency characteristics of cavitation signal by frequency division for cavitation status recognition. The wavelet transform can finely observe the signal detail in time-frequency domain by decomposing the time-domain signals into independent frequency bands on the basis of multi-scale orthogonal refinement comparing with the Fourier transform. However, the wavelet packet transform can further decompose the high-frequency, which cannot be subdivided by the wavelet transform (Yang 2018). Due to the axial passing frequency (APF) and the sampling frequency is respectively $49 \mathrm{~Hz}$ and $5.12 \mathrm{kHz}$, the $\mathrm{db} 4$ basis function is employed to proceed 5-layer wavelet packet decomposition for vibration signals. Then, the cavitation vibration signals are decomposed into 32 frequency bands. That is, the frequency bandwidth of each node is 40 $\mathrm{Hz}$ including APF. The time-frequency of cavitation vibration under different cavitation statuses at rated flow is shown in Fig. 3.

It can be seen from Fig. 3 that the time-frequency domain distribution rules of the five measuring points are similar. In the time domain, the signal periodicity at shaft frequency, blade frequency and frequency multiplication are obvious and amplitude is similar for different periods when cavitation does not occur. The amplitude of vibration acceleration in each frequency band increases, and it is more difficult to recognize the blade passing frequency with the decrease of cavitation number. The amplitude difference of different periods also increases, and chaos amplitude occurs in the highfrequency band which the periodicity is not obvious. It can be seen that cavitation will not only increase the amplitude of vibration acceleration, but also induce the vibration acceleration to periodically deteriorate. In the frequency domain, the amplitude of vibration acceleration appears at the shaft frequency, blade frequency and two-times blade frequency during the non-cavitation status. In the inception cavitation status, the whole frequency band energy increased, the blade frequency and two-times blade frequency reach a maximum. The frequency band began to appear broadband characteristics. In the serious cavitation status, the energy of the whole frequency band increases dramatically, and the broadband amplitude band widens at shaft frequency, blade frequency and twotimes blade frequency. The peak value of shaft frequency and frequency multiplication cannot be distinguished, and large number of discrete distribution amplitudes appear at high frequency.

\subsection{Feature Extraction Based on Frequency} Division Statistics

In order to describe the cavitation energy characteristics of vibration signals more precisely, it is necessary to divide the frequency band of the signal. The appropriateness of frequency band directly determines the accuracy of subsequent feature extraction. The frequency resolution is low if the frequency band is too large. There will be more eigenvalues, and easy redundancy if the frequency band is too small. The WPD subdivides all the nodes into frequency bands too finely, which is not suitable for all the characteristic frequency band analysis. In order to further accurately divide frequency bands, the energy values of each frequency band are calculated and normalized into energy coefficients. The higher the node energy coefficient is, the larger the proportion of the node in the whole signal is. The more cavitation characteristic information it contains. The change curve of energy coefficient under different cavitation number is shown in Fig. 4 at rated flow. 


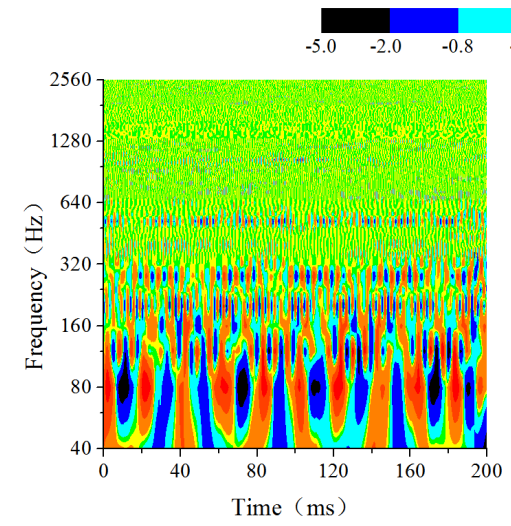

(a1) Non-cavitation $\sigma=0.058$ for the exit

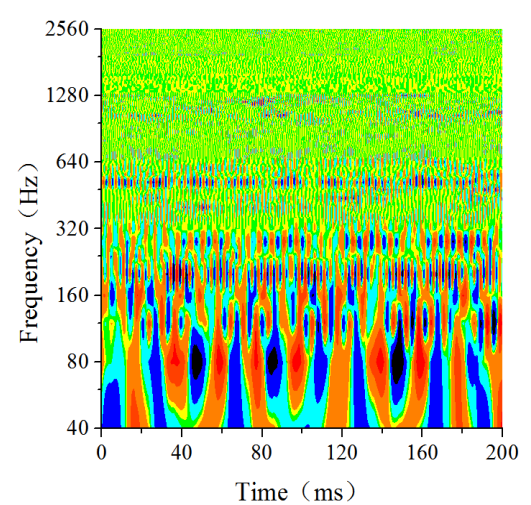

(a2) Inception cavitation $\sigma=0.020$ for the exit

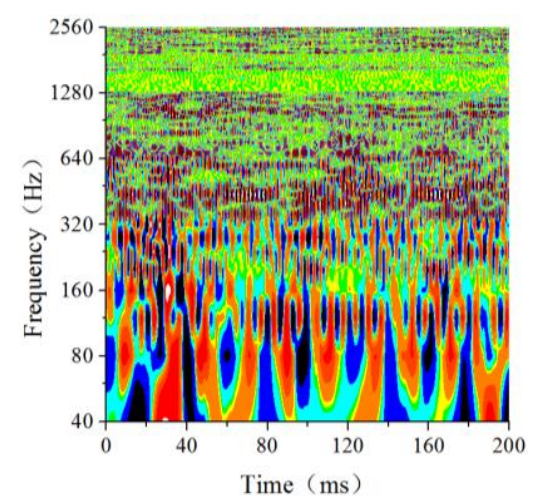

(a3) Serious cavitation $\sigma=0.018$ for the exit

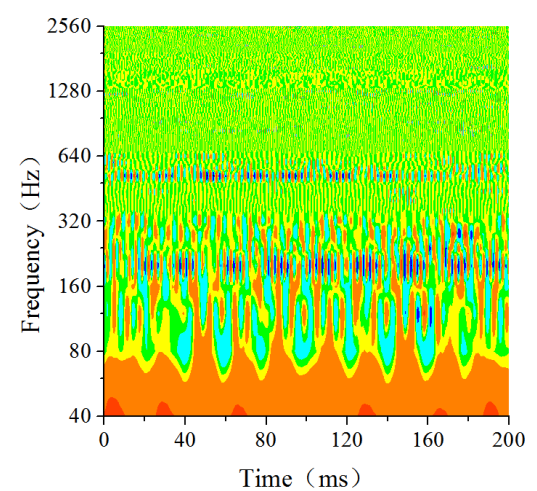

(b1 ) Non-cavitation $\sigma=0.048$ for the pump body

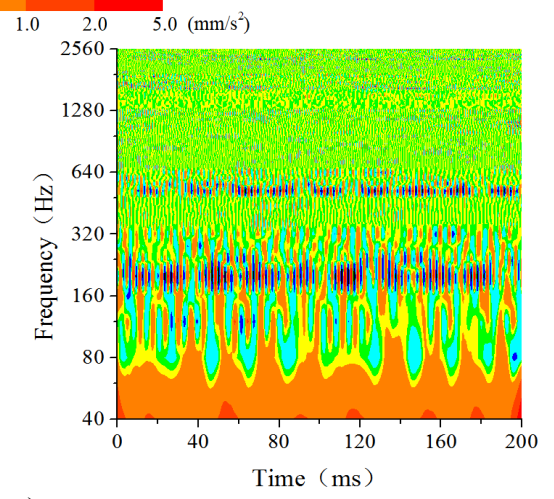

(b2) Inception cavitation $\sigma=0.022$ for pump body

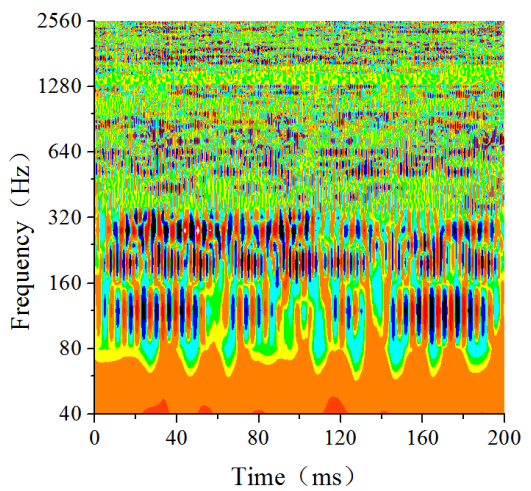

(b3) Serious cavitation $\sigma=0.019$ for pump body

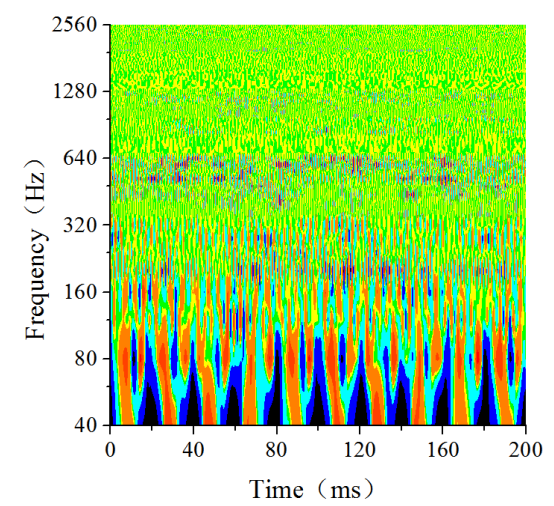

(c1) Non-cavitation $\sigma=0.058$ for the inlet

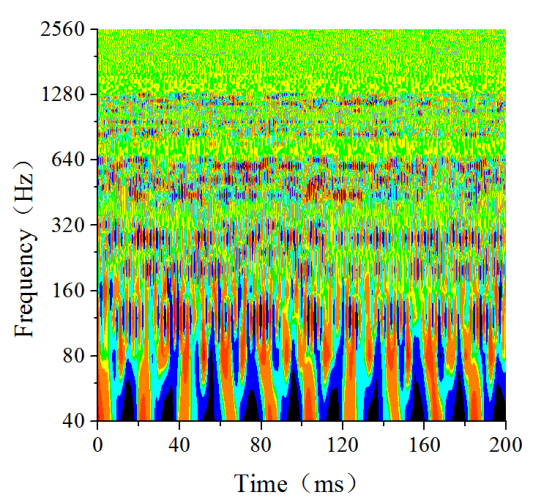

(c2) Inception cavitation $\sigma=0.020$ for the inlet 
L. Dong et al. / JAFM, Vol. 14, No. 1, pp. 315-329, 2021.

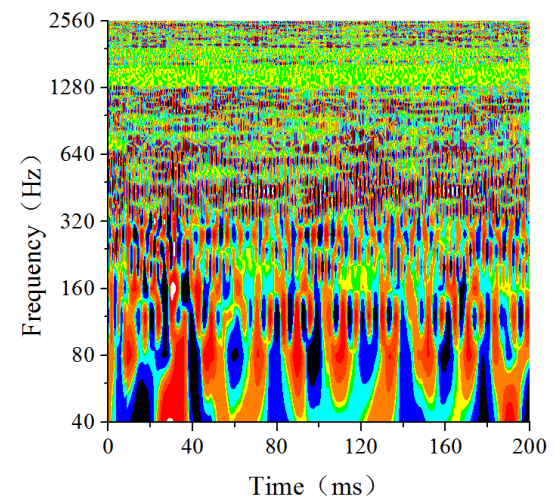

(c3) Serious cavitation $\sigma=0.018$ for the inlet

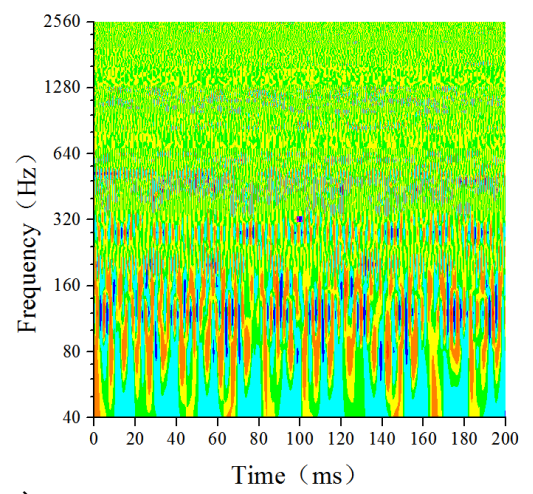

(d1) Non-cavitation $\sigma=0.058$ for the pump foot

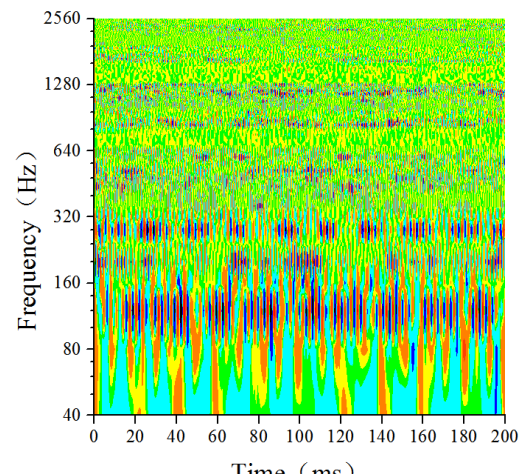

(d2) Inception cavitation $\sigma=0.020$ for the pump foot

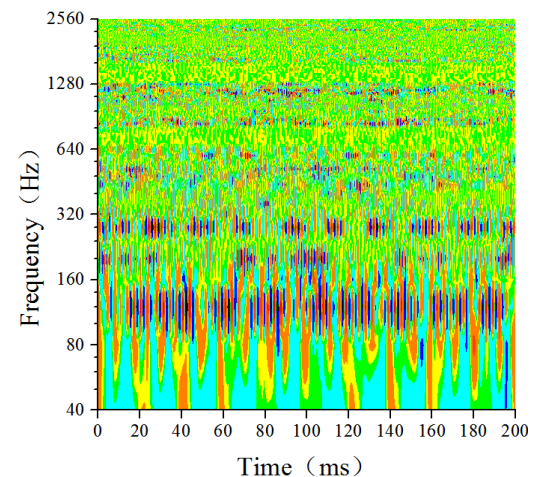

（d3） Serious cavitation $\sigma=0.018$ for the pump foot

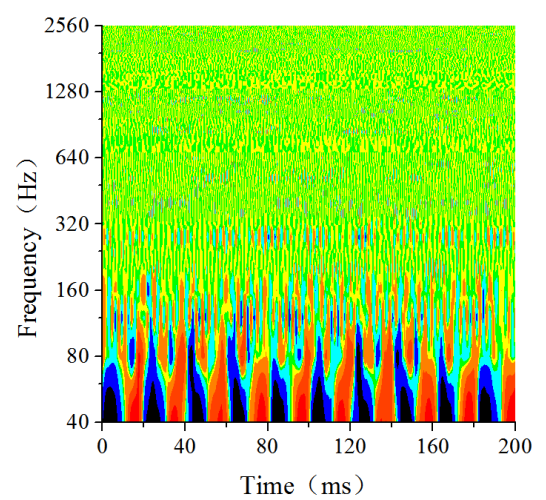

(e1) Non-cavitation $\sigma=0.058$ for the pump cover

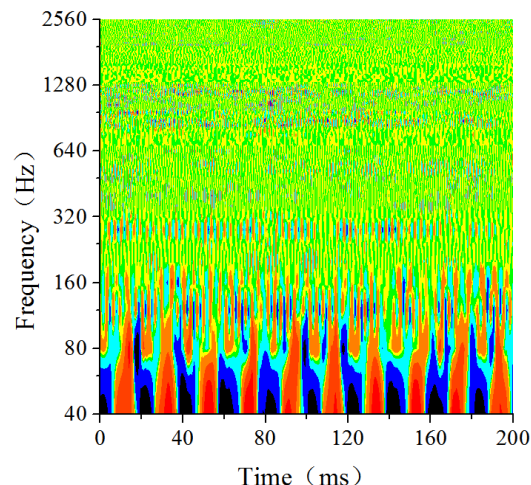

(e2) Inception cavitation $\sigma=0.020$ for the pump cover

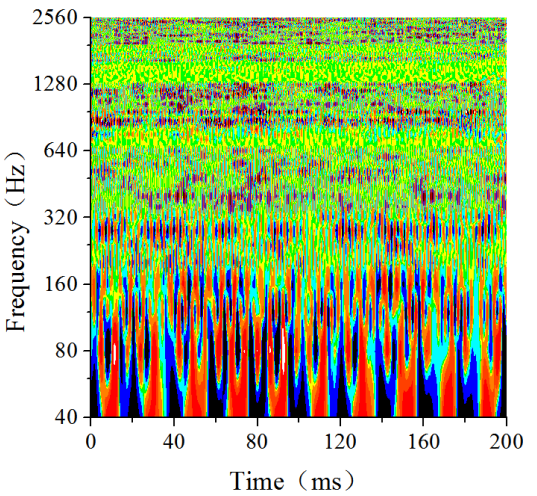

(e3) Serious cavitation $\sigma=0.018$ for pump cover

Fig. 3. Time-frequency domain diagram of cavitation vibration for flow rate. 


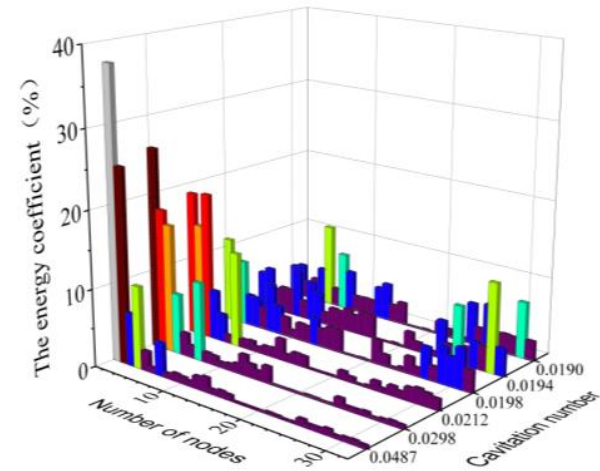

(a) Export flange

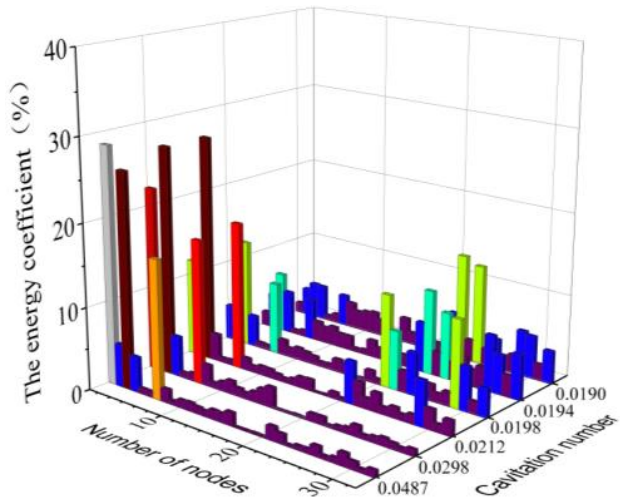

(b) Pump body

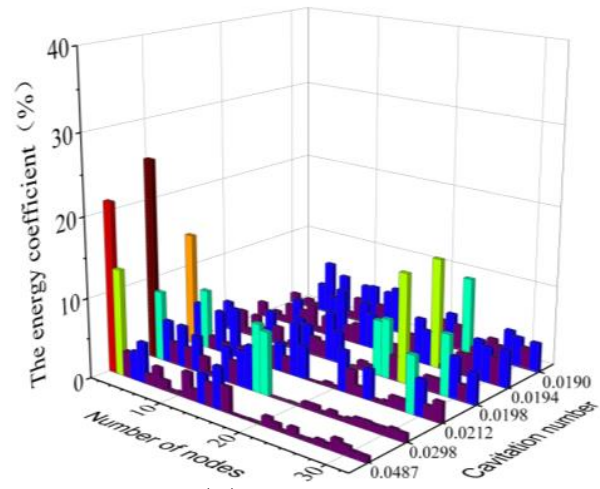

(c) Import flange

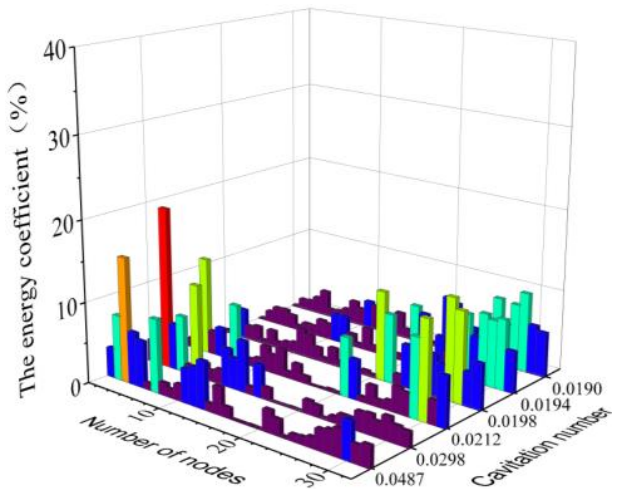

(d) Pump foot

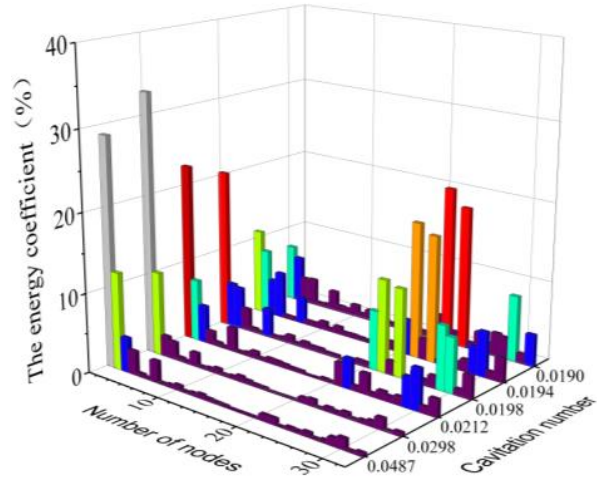

(e) Pump cover

Fig. 4. Energy coefficient under different cavitation numbers at rated flow rate.

As can be seen from Fig. 4, the peak value of signal energy coefficient of each vibration measurement point is mainly focused on the first 6 nodes before cavitation. With the decrease of cavitation number, the vibration signal energy gradually shifts from the low-frequency band to the high-frequency band. The characteristic of broadband peak value appears at the labeled 20-31 nodes. Discrete nodes with large energy coefficients are selected in this paper, and the nodes with small energy proportions are merged to take the upper nodes as the analysis frequency band. Combining Shannon entropy, the optimal wavelet packet decomposition wavelet tree and frequency band corresponding to each node coefficient are obtained, as shown in Fig. 5. Each node at the bottom of the optimal wavelet tree of each sensor is taken as the analysis object, the root mean square value (RMS), standard deviation (STD), mean value (ME), energy value (E), energy coefficient (Ekm) and energy entropy (ES) of each node are calculated respectively to obtain the eigenvalue for recognize cavitation status.

\subsection{Feature Reduction Based on PCA}

Due to large differences during eigenvalue elements, the eigenvalue obtained by the WPD is not conducive for RBF neural network establishment. At the same time 600 sets of vibration acceleration data under different flow conditions were selected, and all eigenvalues were 


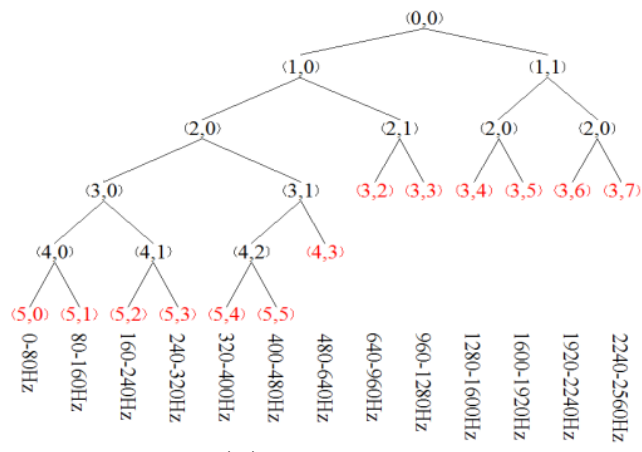

(a) Export flange

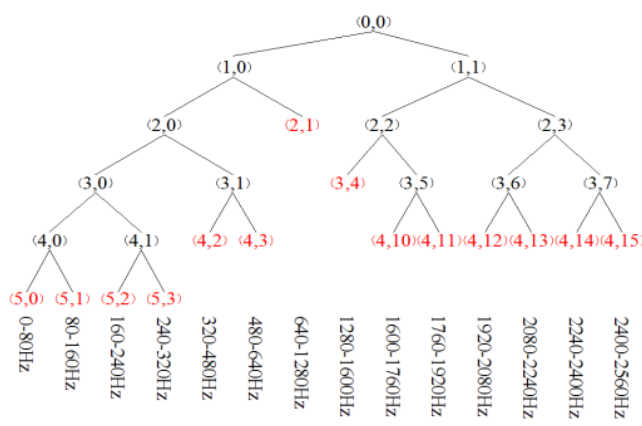

(b) Pump body

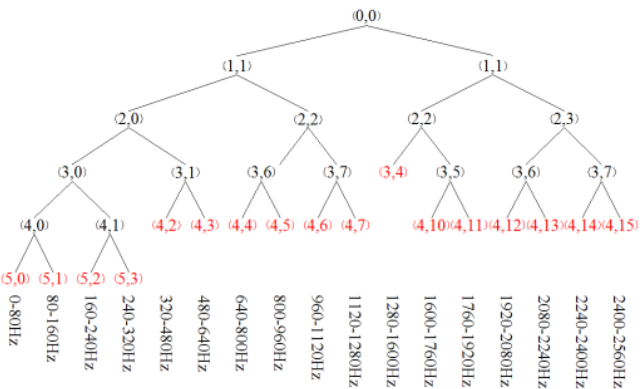

(c) Import flange

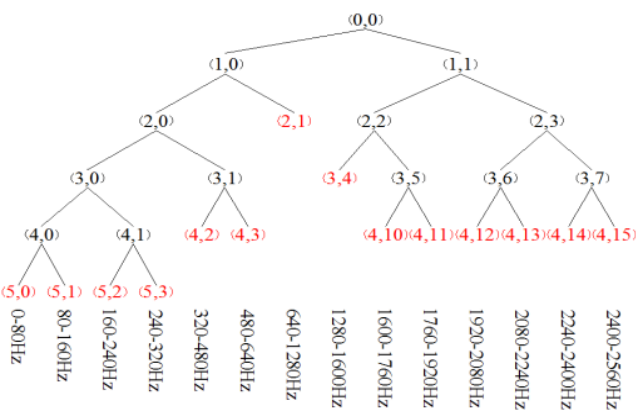

(d) Pump foot

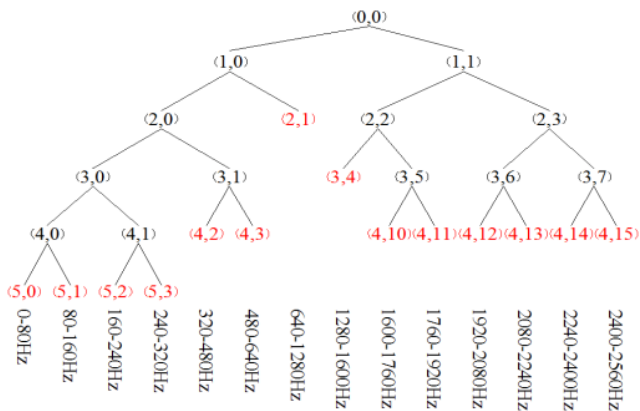

(e) Pump cover

Fig. 5. Optimal wavelet tree and corresponding frequency bands for each node.

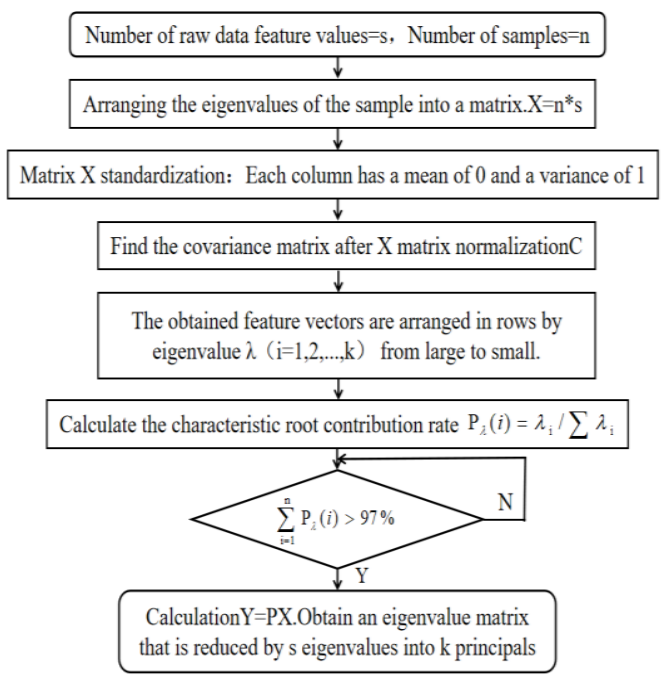

Fig. 6. Flowchart of PCA. prepared for establishing RBF neural network (Su et al. 2017; Liu et al. 2013). The dimensionality reduction was carried out by PCA method, as shown in Fig. 6.

Vibration acceleration data at measuring points of outlet flange, pump body, inlet flange, pump foot and pump cover of a total of 600 groups of samples under different flow conditions are selected. $6 * 13$, $6 * 17,6 * 14,6 * 14$ and $6 * 14$ characteristic values of the 600 groups of sample measurement points are respectively extracted for normalization, and the results are input into the PCA to solve the Eigenvector of covariance matrix. The values are $\lambda$ ${ }_{\text {outlet }}=(158.0151338,112.852235,26.23611328, \ldots$, $0.000257448,0.00024282,0.000127744), \lambda$ ритр ${ }_{\text {body }}=(197.5678488,51.73990437,35.40731719 \ldots$, $0.000152658,0.000128123,0.000110936), \lambda$ inlet $=$ $(186.224213, \quad 56.7362696, \quad 24.99610724 \ldots$ $0.000176659,0.000163181,0.000124848), \lambda$ ритр $f_{\text {oot }}=(235.1375207,65.54817124,21.97917267 \ldots$, 


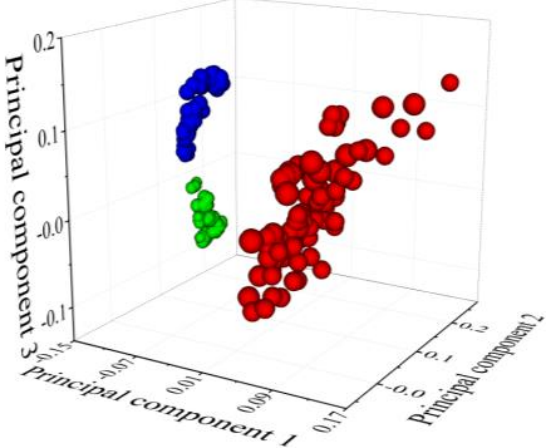

(a) Export flange

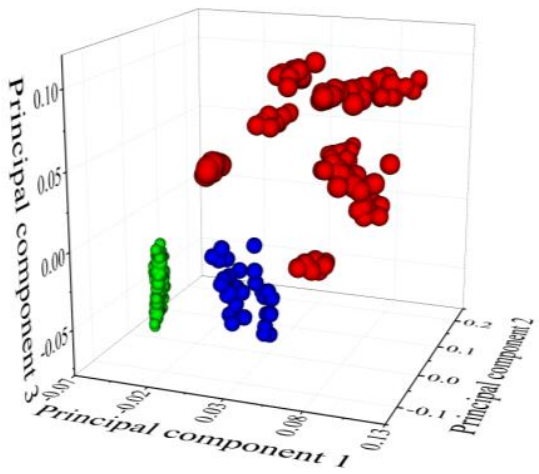

(b) Pump body

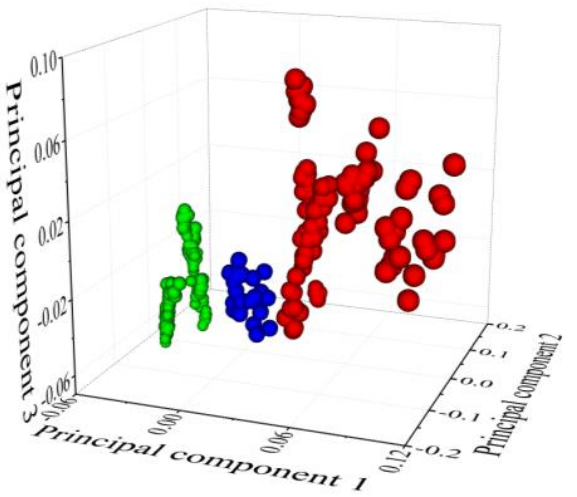

(c) Import flange

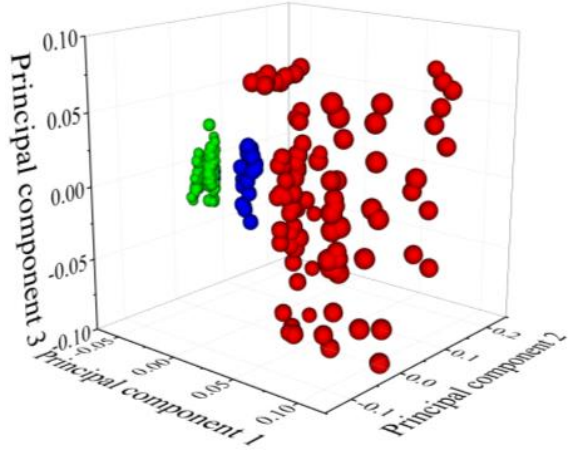

(d) Pump foot

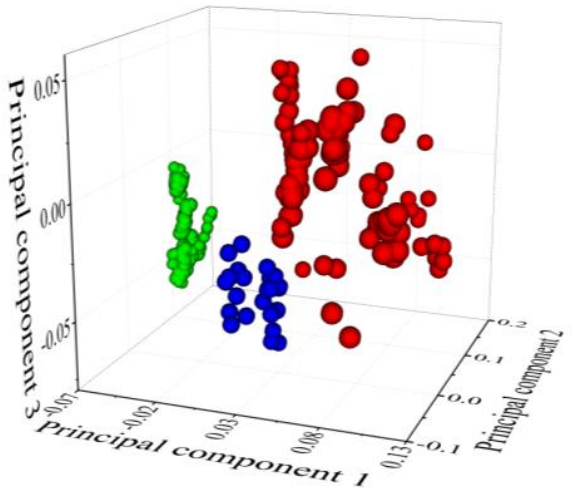

(e) Pump cover

Fig. 7. Space distribution of principal components based on PCA.

$0.00028599, \quad 0.000168031, \quad 0.000124887), \quad \lambda$ ритр cover $=(154.0590444,52.58828974,40.41633536 \ldots$, $0.000213952,0.000180413,0.000136115)$.

The contribution rate of each characteristic root $P_{\lambda}$ is calculated. At present, when the sum of root contribution rate of $\mathrm{n}$ features is greater than $97 \%$, it is considered to be able to better express the characteristics of all the original data. The original sample characteristic matrix was reduced to $600 * n$ principal components. The principal component $n$ was obtained through calculation, namely, the number of input nodes was taken as $20,16,17,18$,
17 and 18 respectively. The PCA based principal element characteristic spatial distribution of each sensor is shown in Fig. 7.

The space distribution of principal components of vibration measuring point is shown in Fig. 7. As can be seen, the bubbles under the status of noncavitation, inception cavitation and serious cavitation are distributed in different spatial regions with no overlap between them. It proves that the principal components obtained by PCA can be used as a good feature for different cavitation statuses. 
Table 2 Input samples and arranged outputs

\begin{tabular}{|c|c|c|c|c|}
\hline Flow rate $/\left(\mathrm{m}^{3} / \mathrm{h}\right)$ & 10 & 12.5 & 15 & Output Result \\
\hline Non-cavitation & 100 & 80 & 100 & $(0,0,1)$ \\
\hline Inception cavitation & 10 & 10 & 10 & $(0,1,0)$ \\
\hline Serious cavitation & 90 & 110 & 90 & $(1,0,0)$ \\
\hline
\end{tabular}

Table 3 Optimal RBF neural network structure parameters of each measurement point

\begin{tabular}{|c|c|c|c|c|}
\hline Measuring point & $\begin{array}{c}\text { Eigenmatrix } \\
\text { dimension }\end{array}$ & Input layer node & Hidden layer node & Output layer node \\
\hline Outlet flange vibration & $6^{*} 13$ & 16 & 10 & 3 \\
\hline Pump body vibration & $6^{*} 17$ & 17 & 10 & 3 \\
\hline Inlet flange vibration & $6^{*} 14$ & 18 & 10 & 3 \\
\hline Foot pump vibration & $6^{*} 14$ & 17 & 10 & 3 \\
\hline Pump cover vibration & $6^{*} 14$ & 18 & 10 & 3 \\
\hline
\end{tabular}

Table 4 Vibration signal RBF neural network process parameters

\begin{tabular}{|c|c|c|c|c|}
\hline Measuring point & $\begin{array}{c}\text { The number } \\
\text { of iterations }\end{array}$ & $\begin{array}{c}\text { The CPU time } \\
(\mathrm{s})\end{array}$ & $\begin{array}{c}\text { The convergence } \\
\text { accuracy }\end{array}$ & $\begin{array}{c}\text { The overall } \\
\text { error }\end{array}$ \\
\hline Outlet flange vibration & 29 & 7.8 & 0.000013 & $98.2 \%$ \\
\hline Pump body vibration & 25 & 7.5 & 0.000051 & $98.3 \%$ \\
\hline Inlet flange vibration & 34 & 8.1 & 0.000006 & $97.8 \%$ \\
\hline Foot pump vibration & 29 & 7.9 & 0.000026 & $97.7 \%$ \\
\hline Pump cover vibration & 37 & 8.3 & 0.000005 & $97.7 \%$ \\
\hline
\end{tabular}

\subsection{Establishment of RBF Neural Network}

The RBF neural network typically has three layers: the input layer, the hidden layer and the output layer. Obviously, the output nodes have been determined, which are non-cavitation status $(0,0$, $1)$, inception cavitation status $(0,1,0)$ and serious cavitation status $(1,0,0)$. In general, the number of hidden layer nodes $\mathrm{m}$ can be firstly calculated as follows (Liao et al. 2015; Chang et al. 2016; Halali et al. (2016):

$\mathrm{m}=\sqrt{n+l}+\alpha$

Where, $n$ is the number of input nodes. $l$ is the number of output nodes. $\alpha$ is the constant between 1 and 10 .

The input samples and the arranged outputs is shown in the Table 2.

After several attempts, the optimal RBF neural network structure parameters of each measurement point are finally determined, as shown in Table 3.

\subsection{One Measurement Point Cavitation Status Recognition}

360 groups of data $(60 \%)$ from 600 samples were selected as the training set. 120 groups of data $(20 \%)$ were regarded as the validation set, and 120 groups of data $(20 \%)$ were regarded as the testing set.

These 600 groups of data were input into the established RBF neural network to obtain the cavitation status recognition results of each measurement point. Due to the randomly selected number of training and testing samples for each time, the obtained recognition rate is different. Process parameters are shown in Table 4. The average recognition results of 10 times are shown in Fig. 8.

According to Fig. 8, the whole recognition rate of all statuses by using the five vibration measurement points is high, which is ranked from the highest to the lowest. Pump body, outlet flange, inlet flange, pump foot, pump cover, respectively $98.3 \%, 98.2 \%$, $97.8 \%, 97.7 \%$ and $97.7 \%$. The recognition rate of non-cavitation status was $83.3 \%, 80.0 \%, 80.0 \%$, $86.7 \%$ and $80.0 \%$, respectively. There is little difference in the recognition rate during vibration measuring points. As shown in Fig. 8, the vibration measurement point at the pump cover is taken as an example. The comprehensive recognition rate of all the statuses at the measurement point of the pump cover is relatively high $(97.7 \%)$, which indicates that the cavitation statuses recognition method combining the vibration signal wavelet packet multi-resolution feature extraction, principal component reduction and RBF neural network is higher precision. The recognition rate of noncavitation status $(100 \%)$ is better than inception cavitation status $(80 \%)$, and all non-cavitation sample data are completely identified. During the 6 groups of samples with wrong judgment, 4 groups are misjudged as non-cavitation and 2 groups are misjudged as serious cavitation, which indicates that the inception cavitation status cannot be completely distinguished from the other two statuses. The recognition rate of serious cavitation 
L. Dong et al. / JAFM, Vol. 14, No. 1, pp. 315-329, 2021.

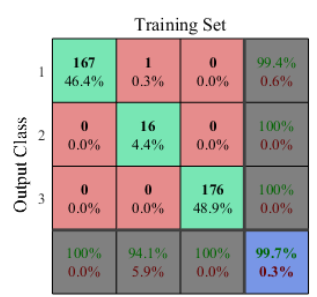

$\stackrel{2}{2} \stackrel{3}{3}$

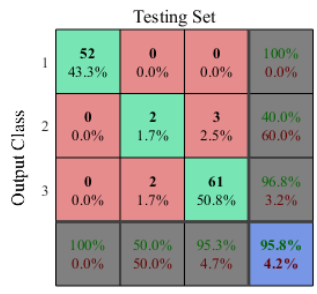

$\stackrel{2}{2} \stackrel{3}{3}$

(a) Export flange

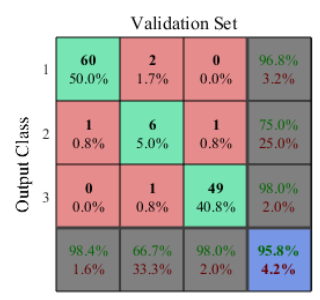

Target Class $^{3}$

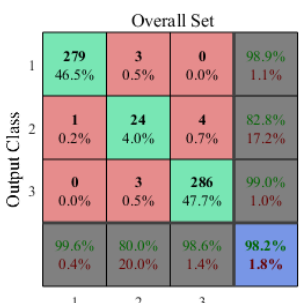

Target Class

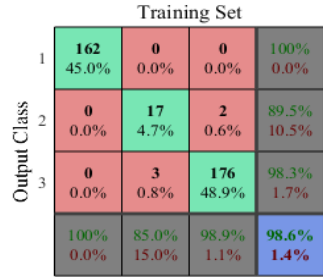

$\stackrel{2}{2}{ }^{3}$

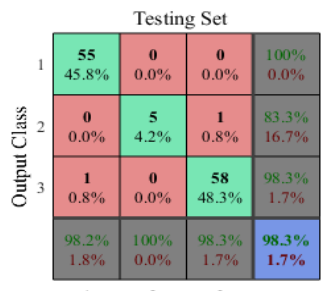

$\stackrel{2}{2}$ Target Clas

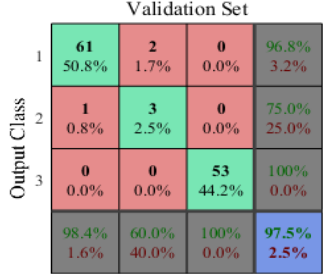

Target Class $^{3}$

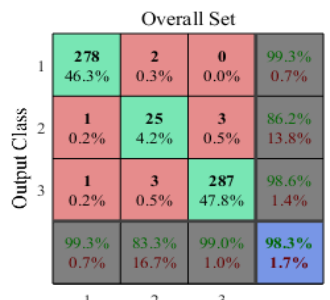

$\stackrel{2}{2}{ }^{3}$

(b) Pump body

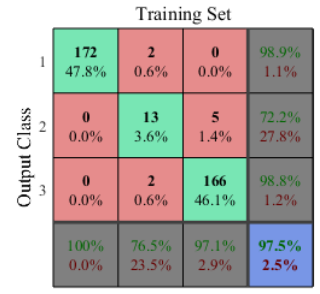

$\stackrel{2}{2}{ }^{3}$

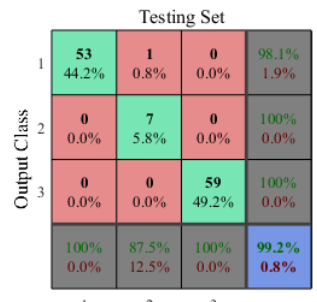

$\stackrel{2}{2} \stackrel{3}{3}$

(e) Pump cover

Fig. 8. RBF neural network recognizes cavitation results. 
status is good, $97.2 \%$, and 8 of the 290 samples data groups are wrongly diagnosed as inception cavitation, which indicates that the distinction between serious cavitation status and non-cavitation status is good, and the distinction between serious cavitation status and inception cavitation status needs to further improve. From the perspective of training set, the recognition rate of the three statuses used for training is $100 \%, 76.5 \%$ and $97.1 \%$ respectively. This indicates that the eigenvalue as the input of the RBF neural network can well reflect the difference between the vibration signal in cavitation and non-cavitation status, but the difference between the signal characteristic in the inception cavitation status and the serious cavitation status is not obvious enough.

Due to the external adverse environment or changes in incentives and abrupt changes in working conditions and other factors, which cause the sensor's acquisition of data is often subject to unpredictable disturbances. The phenomenon of mutation or deletion of original data occurs from time to time. In order to study the ability of a single measure point cavitation status recognition method to resist unknown disturbances, white noise is taken as the interference signal, and the ratio of the interference signal length to the whole signal length is defined as the interference coefficient $\theta$. The interference signal was implanted into the test sample signal, and the interference coefficient was increased by $5 \%$ as a measure to conduct the simulation test, and the anti-interference performance of cavitation recognition at different measuring points was obtained as shown in Fig. 9.

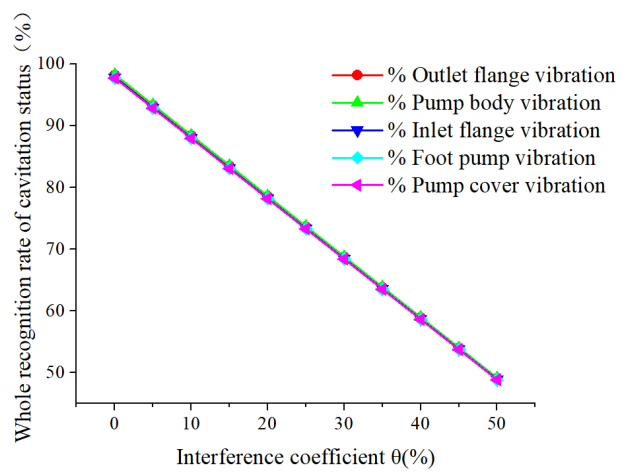

Fig. 9. Single measuring point anti-interference.

It can be seen from Fig. 9 that the cavitation status recognition rate using one measurement point almost decreases linearly with the increase of the interference coefficient. Because the number of effective test samples of one measurement point decreases with the addition of the interference signal. The recognition rate of the invalid test sample is basically zero, which leads to a very rapid decline in the whole identification rate of the cavitation status, and the difference in the antiinterference of the different measurement points is not obvious.

\subsection{Cavitation Status Recognition at Multiple Measuring Points}

It can be seen that the whole recognition rate of cavitation status recognize method using single measurement point is good, but the recognition rate of the most concerned inception cavitation status is poor when one measurement point is used for cavitation status recognition. Once the sensor signal is interfered by the external environment, the recognition result becomes worse. In order to further improve the inception cavitation status recognition rate and anti-interference, six eigenvalues of each node are extracted for the same 600 groups of samples for each measuring point, and the eigenvalues of these measuring points are divided into different measuring points and combinations. The new characteristic matrix arranged, and the PCA is used to reduce the dimension and then trained into a new different RBF neural network to achieve information fusion. The multi-measurement signal information fusion cavitation status recognition result is shown in Fig. 10

It can be seen from Fig. 10 that the information fusion of multiple measurement points is significantly better than one measurement point for three cavitation statuses recognition. The more measurement points are, the higher the recognition rate is. The non-cavitation status and the serious cavitation status are close to $100 \%$ when the information fusion of two measuring points is used for status recognition. The recognition rate of the serious cavitation status is higher than one measuring point, and the partial combination of the recognition rate of the inception cavitation status is improved. The different combination methods of information fusion status recognition of two measuring points are compared and analyzed. The results show that the vibration measurement points at the pump body or the outlet flange have the highest recognition rate, and the following is the vibration measuring point including the inlet flange, the pump foot or the pump cover. When any three or more measuring points are used for information fusion, the recognition rate of three cavitation statuses is $100 \%$ regardless of which combination of measuring points is adopted. And it shows that the multi-point signal fusion method can accurately and effectively recognize different cavitation statuses.

Due to external excitation changes and sudden changes in operating conditions, making the sensor acquire data often suffers from unpredictable disturbances. In order to study the anti-interference of multi-point information fusion method based on RBF neural network in cavitation status recognition. Taking the five measuring point fusion as an example, the original signal value of the sensor at the four of the five measuring points of the pump body, outlet flange, inlet flange, pump foot and pump cover is kept unchanged. With the remaining one measuring point as the interference item, the interference coefficient of the interference item was adjusted and added into the interference item as a 


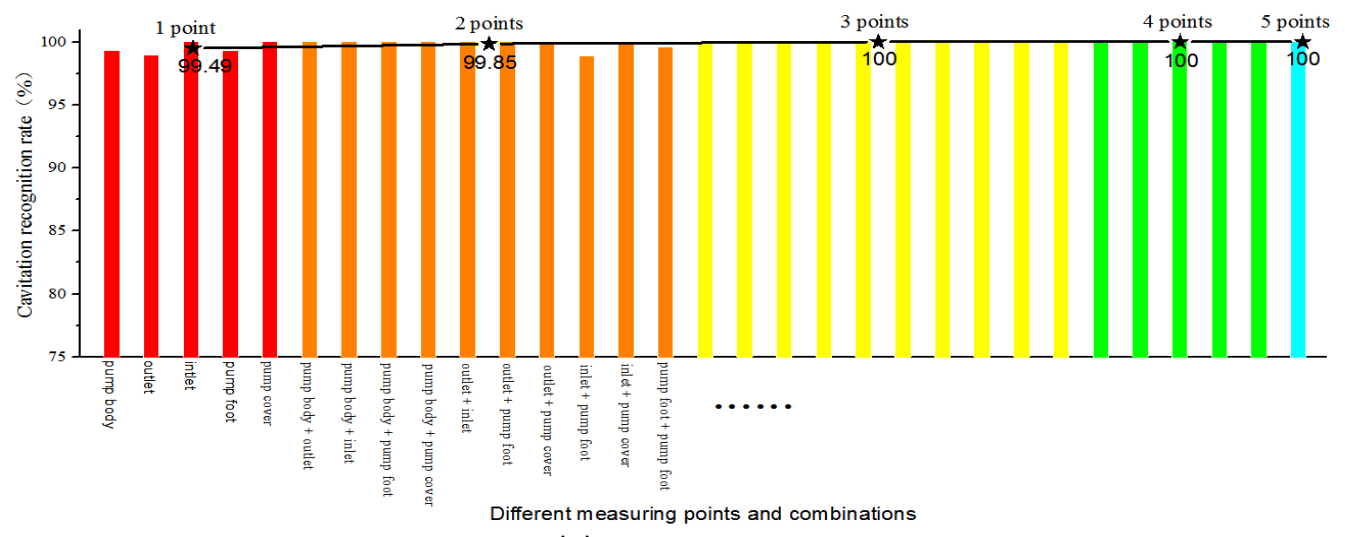

(a) Non-cavitation

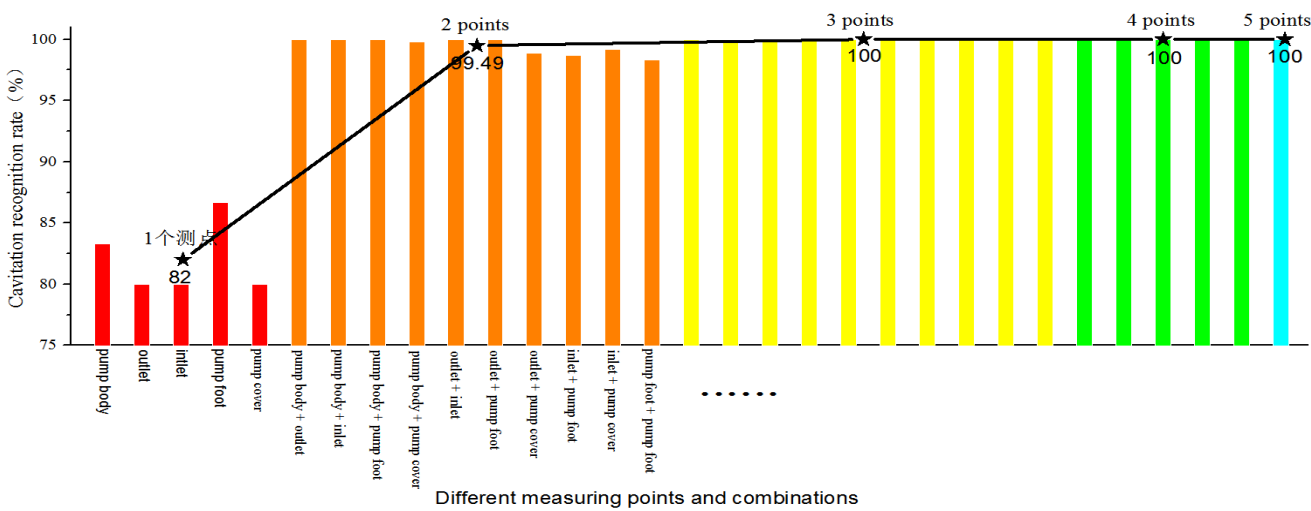

(b) Inception cavitation

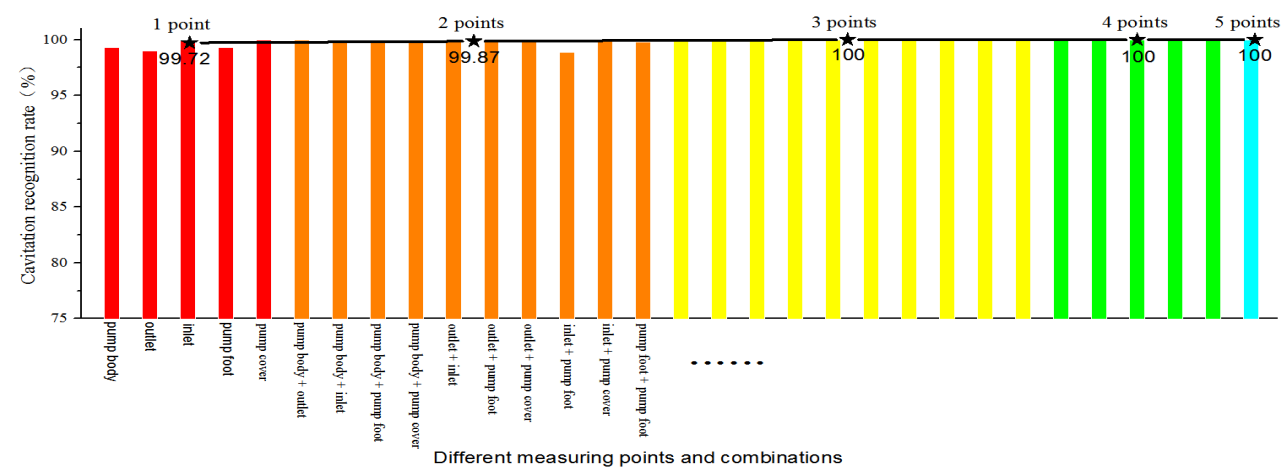

(c) Serious cavitation

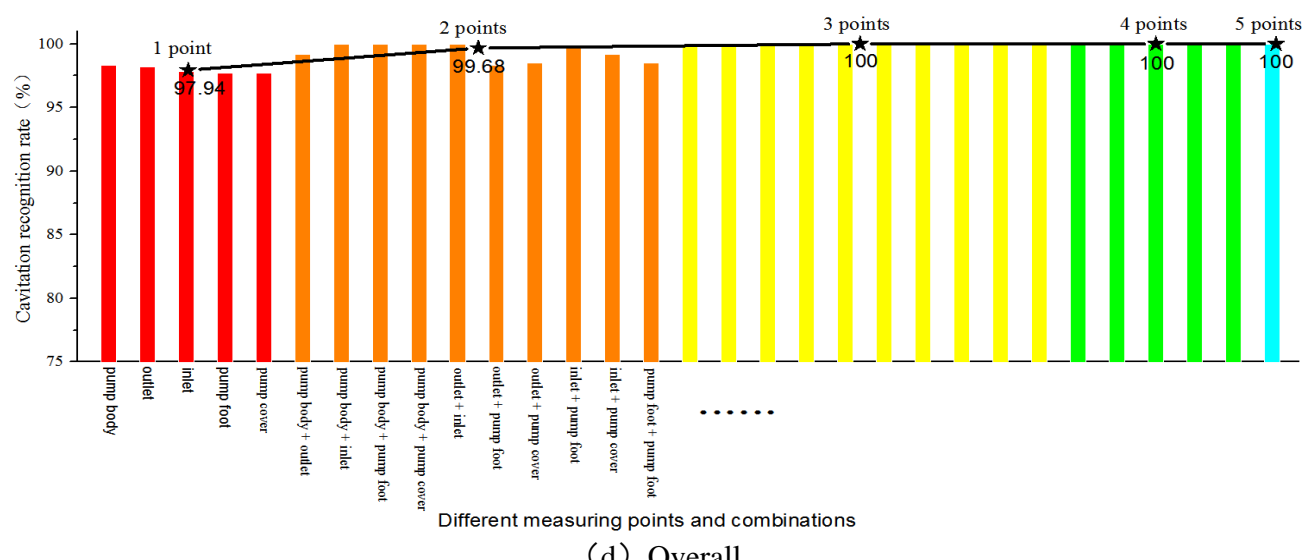

Fig. 10. Multi-measurement information fusion cavitation status recognition result. 
measure with the interference coefficient increased by $5 \%$. Simulation experiment was conducted. The number of interference items is adjusted to $2-4$, and the recognition rate under different interference conditions is shown in Fig. 11.

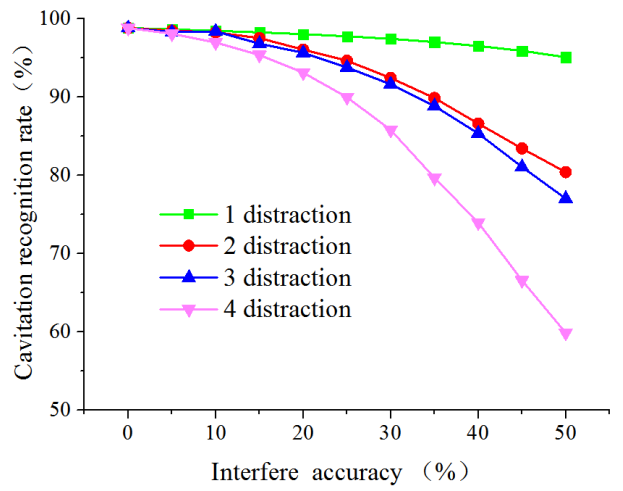

Fig. 11. Anti-interference of information fusion method based on RBF neural network.

It can be seen from Fig. 11, the recognition rate of the cavitation status of the multi-point fusion decreases gradually with the enhancement of the interference, and decreases faster and faster. The higher the interference is, the lower recognition rate of cavitation status and the faster the decrease is. The interference degree has little influence on the status recognition result when there is only one interference item. When the interference coefficient drops by $50 \%$, the cavitation status recognition rate reaches more than $80 \%$, which is obviously better than the one measurement point recognition result. However, when there are more than two interference items, the status recognition rate decreases rapidly with the increase of interference coefficient. In the case of interference in four of the five measuring points, the recognition rate is more than $80 \%$ when the interference coefficient is less than $30 \%$. When the interference coefficient is $50 \%$, the recognition rate is lower than $60 \%$. Therefore, the multi-point information fusion method based on RBF neural network can effectively be improved the anti-interference performance of cavitation status recognition. However, when there are many interference items and the interference coefficient is large, the recognition rate also declines seriously.

\section{Conclusion}

A multi-resolution cavitation status recognition method of one measurement point of centrifugal pump is proposed based on the vibration signal, which is independent of the working condition. The analysis results show that the whole recognition rate of the three cavitation statuses of non-cavitation, inception cavitation and serious cavitation is more than $97.7 \%$, and the recognition rate of the inception cavitation is more than $80 \%$.

In order to solve the problem of low recognition rate of inception cavitation status by multiresolution analysis method of single measuring point signal, a cavitation status recognition method based on multi-measuring point information fusion is proposed. The eigenvalue of each node of single measurement point is arranged into a new characteristic matrix according to the number and combination of different measurement points, and the new RBF neural network is trained after the PCA is adopted to realize information fusion. The analysis results show that the recognition rate of inception cavitation status based on the two-point signal information fusion method is more than $99 \%$, and the recognition rate of three kinds of cavitation statuses based on the three-point signal recognition fusion method is all up to $100 \%$.

The anti-interference performance of the proposed cavitation status recognition method is analyzed. The results show that when there is only one interference term among the five measuring points and the interference coefficient is $50 \%$, the cavitation status recognition rate is still above $90 \%$. In the case where there are interferences in four of the five measuring points, the recognition rate is above $80 \%$ when the interference coefficient is within $30 \%$. When the interference coefficient is $50 \%$, the recognition rate is as low as $60 \%$ or less.

\section{ACKNOWLEDGEMENTS}

This work was supported by National Key Research and Development Program of China (Grant No 2016YFB0200901, 2017YFC0804107), National Natural Science Foundation of China (No. 51879122, 51579117, 51779106), Zhenjiang key research and development plan (GY2017001, GY2018025), the Open Research Subject of Key Laboratory of Fluid and Power Machinery, Ministry of Education, Xihua University (szjj2017-094), Sichuan Provincial Key Lab of Process Equipment and Control (GK201614, GK201816), the Advanced Talent Foundation of Jiangsu University (15JDG052) and a project funded by the Priority Academic Program Development of Jiangsu Higher Education Institutions (PAPD).

\section{REFERENCES}

Alfayez, L. Mba, D. Dyson, G (2005). The application of acoustic emission for detecting incipient cavitation and the best efficiency point of a $60 \mathrm{~kW}$ centrifugal pump: case study [J]. NDT\&E International 38(5), 354-358.

Azizi, R., B. Attaran, A. Hajnayeb, A. Ghanbarzadeh and M. Changizian (2017). Improving accuracy of cavitation severity detection in centrifugal pumps using a hybrid feature selection technique. Measurement 108, 9-17.

Bourdon, P., M. Farhat, Y. Mossoba and P. Lavigne (1999). Waterpower'99: Hydro's Future: Technology, Markets, and Policy. American Society of Civil Engineers, Reston, American.

Chang, S, N. M. Leng, Y. H. Wu, W. J. Thompson (2016). Aircraft ice accretion prediction using 
neural network and wavelet packet transform, Aircraft Engineering and Aerospace Technology 88(1), 128-136.

Dong, L., K. Wu, J. Zhu, C. C. Dai (2019). Cavitation Detection in Centrifugal Pump Based on Interior Flow-Borne Noise Using WPD-PCA-RBF. Shock and Vibration 2019.

Dong, L., Y. Zhao, Q. C. Dai (2019). Detection of inception cavitation in centrifugal pump by fluid-borne noise diagnostic. Shock and Vibration.

Durocher, D. and B. G. R. Feldmeier (2004). Predictive versus preventive maintenance. IEEE Industry Applications Magazine 10(5), $12-21$.

Escaler, X., E. Egusquiza, T. Mebarki and F. Avellan (2002, September). Field Assessment of Cavitation Detection Methods in Hydropower Plants, Proceedings of the Hydraulic Machinery and Systems 21st IAHR Symposium, Lausanne, Switzerland, 1(CONF), 483-490. Association For Hydraulic Research.

Farokhzad, S. and H. Ahmadi (2013). Acoustic based cavitation detection of centrifugal pump by neural network. Journal of Mechanical Engineering and Technology.

Halali, M, A. Azari, V. Arabloo and M. A. H. Mohammadi (2016). Application of a radial basis function neural network to estimate pressure gradient in water-oil pipelines. Journal of the Taiwan Institute of Chemical Engineers (58), 189-202.

He, G., Y. Cao, T. Ming and Y. Su (2017). Cavitation state recognition of centrifugal pump based on features of modified octave bands. Journal of Harbin Engineering University 38(8), 1263-1267 and 1302.

Hernandez-Solis, A. and F. Carlsson (2010). Diagnosis of submersible centrifugal pumps: a motor current and power signature approaches. European Power Electronics and Drives Journal 20(1), 58-64.

Johann, F. G. (2014). Centrifugal pump. Heidelberg: Springer, Berlin, Germany.

Liao, G., L. Du, L. Su and L. M. Zeng (2015). Using RBF networks for detection and prediction of flip chip with missing bumps. Microelectronics Reliability (55), 2817-2825.

Liu, M., H. Nan and X. Yu (2013). Fault diagnosis of hydro turbine regulation system based on PCA-SDG. Journal of Drainage and Irrigation Machinery Engineering 31(12), 1065-1071.

Panda, A. K., J. S. Rapur and R Tiwari (2018). Prediction of flow blockages and impending cavitation in centrifugal pumps using support vector machine (SVM) algorithms based on vibration measurements. Measurement 130, 44-56.

Rus, T., M. Dular, M. Hocevar, S. Brane, H. Marko and K. Igor (2007). An investigation of the relationship between acoustic emission, vibration, noise and cavitation structures on a kaplan turbine. Journal of Fluids Engineering 129(9), 1112-1122.

Sakthivel, N, R. Nair, B. B. Elangovan, M. Sugumaran and V. S. Saravanmurugan (2014). Comparison of dimensionality reduction techniques for the fault diagnosis of mono block centrifugal pump using vibration signals. Engineering Science and Technology 17, 3038

Samanipour, P., J. Poshtan and H. Sadeghi (2017). Cavitation detection in centrifugal pumps using pressure time-domain features. Turkish Journal of Electrical Engineering \& Computer Sciences 25(5), 4287-4298.

Su, L., T. Shi, L. Z. Liu, and P. H., D. Zhou (2017). Nondestructive diagnosis of flip chips based on vibration analysis using PCA-RBF. Mechanical Systems and Signal Processing 85, 849-856.

Wang, H. and P. Chen (2009). Intelligent diagnosis method for a centrifugal pump using features of vibration signals. Neural Computing and Applications 18(4), 397-405.

Xue, H., K. Li, H. Wang and P. Chen (2013, July). Sequential diagnosis method for rotating machinery using support vector machines and possibility theory. Proceedings of the International Conference on Intelligent Computing, Heidelberg, Berlin, Germany, 315324. Springer.

Xue, Y. G. (2016). An investigation into fault diagnosis of hydro-turbine unit based on EMD multifractal spectrum. Journal of Drainage and Irrigation Machinery Engineering 34(5), $455-460$

Yagi, Y., M. Murase, K. Sato and Y. Saito (2003). Preliminary study of detecting the occurrence of cavitation and evaluating TTS influence by an accelerometer mounted on a pipe. Fifth International Symposium on Cavitation, Osaka, Japan, 2003.

Yang, X. G. (2018). Pattern recognition of engine abnormal sound based on wavelet packet and bispectrum. Noise and Vibration Control 38(6), 146 